H. C. Hughes

\title{
Effects of warning signals and fixation point offsets on the latencies of pro- versus antisaccades: implications for an interpretation of the gap effect
}

\begin{abstract}
The present study was designed to evaluate whether fixation point offsets have the same effects on the average latencies of prosaccades (responses towards target) and antisaccades (responses away from target). Gap and overlap conditions were run with and without an acoustic warning signal. The 'gap effect' was taken to be the difference in mean reaction time between gap and overlap trials. This effect was dramatically reduced by the presentation of the warning signal. Without this signal, fixation offsets can serve as warning signals themselves, which artifactually inflates the magnitude of the gap effect. The warning effect of fixation offsets was equivalent for pro and antisaccades. A significant gap effect is still evident with the acoustic warning signal; however, in this case it is associated primarily with prosaccades. These results replicate and extend our previous work demonstrating that, if their warning effects are controlled, the facilitatory effects of fixation point offsets are response dependent, and suggesting the existence of a component process (fixation release) which is closely linked with the processing architecture underlying target-directed saccades.
\end{abstract}

Key words Gap effect - Express saccades - Oculomotor Human

Portions of this work were presented at the annual meeting of the Association for Research on Vision and Ophthalmology, Sarasoto, 1993

P. A. Reuter-Lorenz ( L. L. Barnes

Department of Psychology, University of Michigan,

525 East University, Ann Arbor, MI 48109-1109, USA;

e-mail: parl@umich.edu

H. M. Oonk

Department of Psychology, University of Washington,

St. Louis, MO 63130, USA

H. C. Hughes

Department of Psychology, Dartmouth College,

Hanover, NH 03755, USA

\section{Introduction}

The effect of fixation point offsets on oculomotor performance has recently become a subject of great interest and debate (e.g., Fischer and Weber 1993). In what has come to be known as the gap paradigm, the fixation stimulus is extinguished prior to the onset of an eccentric saccade target. Numerous reports indicate that this simple manipulation can produce dramatic reductions in saccadic latencies. In the original investigation of this effect, Saslow (1967) reported a significant reduction in mean saccadic latency in the gap condition relative to an overlap condition in which the fixation stimulus remains visible while the target is presented. The optimal gap duration was reported to be approximately $200-300 \mathrm{~ms}$.

Inspired by the work of Fischer and his colleagues (Fischer and Boch 1983; Fischer and Breitmeyer 1987; Fischer and Weber 1992), more recent interest in the gap paradigm has focused on the occurrence of express saccades rather than on an analysis of mean saccadic latency. Fischer and Ramsperger $(1984,1986)$ originally identified express saccades in humans as a subpopulation of rapid responses with latencies ranging from 80 to $120 \mathrm{~ms}$. Express saccades are reported to have a modal latency of $100 \mathrm{~ms}$, which constitutes the early mode in a bimodal (or multimodal) distribution of saccade latencies.

However, for many subjects it is difficult to discern evidence of bimodality or multimodality in their latency distributions. In some reports, $40 \%$ of subjects fail to show this effect (Fischer et al. 1993), whereas in other reports the estimate is much higher (Reuter-Lorenz et al. 1991; Wenban-Smith and Findlay 1991; Kingstone and Klein 1993b) While many investigators agree that saccades in the 100-120 ms range occur in the gap condition, the identification and the quantification of a reliable subpopulation of such responses has proven to be problematic (see Kingstone and Klein 1993b, Klein and Kingstone 1993 for discussion; Reuter-Lorenz and Hughes 1993; Nozawa et al. 1995). Nevertheless, even those subjects who do not demonstrate clear evidence of "express 
saccades" show a dramatic reduction in saccadic latency in the gap condition relative to the overlap condition. This reduction in mean saccadic latency has been referred to as the "gap effect" (e.g., Reuter-Lorenz et al. 1991).

The effect of the gap paradigm has captured the interest of eye movement researchers because of the possibility that it reveals facilitatory processes that are specific to the oculomotor system. In particular, a number of researchers have suggested that the gap paradigm may reveal the operation of a reflexive, collicular-based oculomotor pathway (e.g., Fischer and Breitmeyer 1987; Schiller et al. 1987; Reuter-Lorenz et al. 1991; Nozawa et al. 1995).

An interpretation of the gap effect and express saccades in terms of facilitatory oculomotor processes depends in part on controlling other nonspecific effects that are associated with fixation point offsets. Ross and Ross $(1980,1981)$ suggested that visual stimulus offsets can serve as effective warning signals producing a reduction in saccadic latencies through a generalized alerting effect. Thus, saccadic facilitation in the gap paradigm could be due, in part, to the alerting effect of fixation point offsets. In fact, Kingstone and Klein (1993a) hypothesize that the gap effect involves two components: one involving generalized response preparation and one specifically involving "fixation release". It is presumed that only this latter effect is specifically associated with oculomotor processes that may depend on the superior colliculus.

To reduce the contribution of the warning effect of fixation offsets, Reuter-Lorenz et al. (1991) included an acoustic warning signal which offset $200-300 \mathrm{~ms}$ prior to target onset in both the gap and overlap conditions. They compared choice manual responses to two types of oculomotor responses: prosaccades, in which subjects fixate an eccentric target, and antisaccades, in which subjects saccade in the direction opposite to an eccentric target. Only prosaccades showed a significant benefit from fixation point offsets. This outcome is consistent with the idea that fixation point offsets can have the specific effect of facilitating the generation of saccades via a rapid, reflexive, oculomotor pathway.

A different outcome has recently been reported by $\mathrm{Fi}$ scher and Weber (1992). Their analysis of antisaccades and prosaccades in the gap paradigm revealed that both responses showed a dramatic latency reduction in the gap condition, i.e., greater than $30 \mathrm{~ms}$ advantage in the gap paradigm. Only prosaccades, however, showed a subpopulation that met their criteria for express saccades.

In the present study, we investigate the possible basis for the different outcomes reported by Fischer and Weber (1992) and Reuter-Lorenz et al. (1991). One notable methodological difference between these studies is that Reuter-Lorenz et al. (1991) included an acoustic warning signal designed to equate response readiness in the gap and overlap conditions whereas Fischer and Weber (1992) did not. In the absence of a warning signal, all types of responses are likely to be faster in the gap condition relative to the overlap condition, since the fixation offsets alert the observer to the imminent occurrence of the target event. Along with Kingstone and Klein (1993a), we suspected that the magnitude of the gap effect and the degree of response specificity would be determined by whether or not a warning signal wass included in the design. The present experiment was designed explicitly to test this prediction.

At issue here is the question of whether or not differences in mean saccadic latencies (what we are calling the "gap effect") provide a useful measure to investigate the facilitatory effects of fixation offsets and the degree to which these effects are response dependent. Fischer and Weber (1993) suggest that the gap effect is not a suitable alternative to the measurement of express saccades because, in their view, fixation offsets influence all types of visual-motor processing, but the reflex-like optomotor response is only revealed by the presence of distinct modes in the (pro)saccadic reaction time (RT) distribution. As we have argued elsewhere (Reuter-Lorenz et al. 1991; Reuter-Lorenz and Hughes 1993), the gap effect is a more reliable measure than express saccades (see also Kingstone and Klein 1993b). Under well controlled conditions, an analysis of mean saccadic latency is an effective measure that, in conjunction with specific models and analytic techniques, can provide important clues concerning the processing architecture underlying both the gap effect and express saccades (e.g., Nozawa et al. 1995).

\section{Materials and methods}

Apparatus

The experiment was conducted in a completely darkened, soundattenuated chamber. Three computer-controlled, red-light-emitting diodes (LEDs) were viewed from a distance of $57 \mathrm{~cm}$ and subtended $0.5^{\circ}$ visual angle. The center LED served as the fixation point and the two peripheral LEDs served as the targets. The target LEDs were positioned $6^{\circ}$ to the right and left of the fixation light. Target Iuminance was set at $0.3 \mathrm{ftc}$. In the warning signal condition, a $50 \mathrm{~dB} 1000 \mathrm{~Hz}$ tone $100 \mathrm{~ms}$ in duration was presented via a $4 \mathrm{~cm}$ speaker positioned directly below the fixation point LED. The position of the left eye was monitored using the Eye-trac 210 infrared scleral reflection device, which has a resolution of $0.25^{\circ}$. The eye position signal was digitized at a sampling rate of $500 \mathrm{~Hz}$. The subject's head movements were minimized with a chin rest.

\section{Subjects}

Ten subjects participated in this experiment: four paid University of Michigan undergraduates, three volunteers from our laboratory, and three of the authors (P.R.L., H.M.O., and L.L.B.). All observers had normal vision or vision corrected by contact lenses.

\section{Design and procedure}

Half of the trial blocks were run with a warning signal and half were run without a warning signal. In separate trial blocks, subjects performed either pro- or antisaccades. Gap and overlap trials occurred randomly, but with equal frequency in each block of 100 
Fig. 1 Schematic representation of gap and overlap conditions with and without an acoustic warning signal

\section{OVERLAP CONDITION}

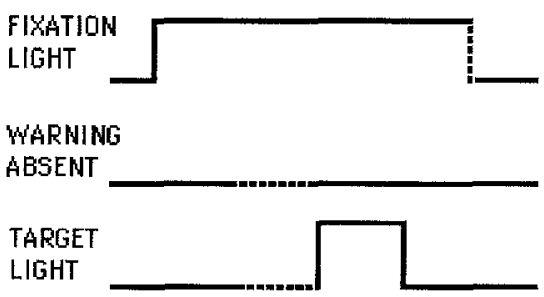

GAP CONDITION

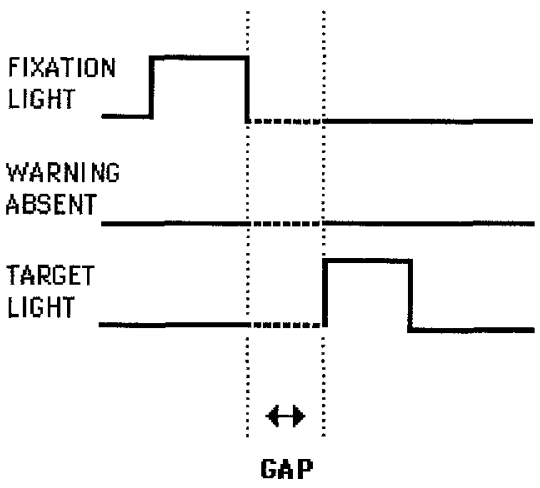

$200-300 \mathrm{~ms}$

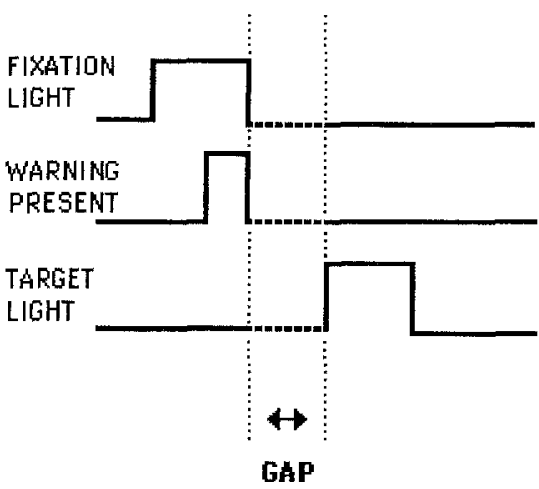

$200-300 \mathrm{~ms}$ trials. A total of eight experimental blocks were run in a fixed order for all subjects. Subjects began with prosaccade blocks, first with warning and then without. Antisaccade blocks followed, again with warning blocks first. Each subject participated in a practice block of 100 trials prior to each new condition. No more than six trial blocks were run in each experimental session, which lasted about $1 \mathrm{~h}$.

The position of the target (either to the left or the right of fixation) was randomized throughout the blocks to prevent anticipatory responses. In addition, $15 \%$ of trials were catch trials in which no target was presented. In a further effort to reduce anticipations, gap durations (and foreperiod durations in the warning-overlap conditions) varied between 200 and $300 \mathrm{~ms}$, randomly assuming any one of five fixed values in $25 \mathrm{~ms}$ steps (i.e., $200,225,250 \mathrm{~ms}$ etc.). The effect of gap duration was not analyzed, however, since previous research from our lab has found equivalent effects from 200 and $300 \mathrm{~ms}$ gaps (Reuter-Lorenz et al. 1991).

A schematic illustration of the trial events is presented in Fig. 1. Each trial began with the illumination of the fixation light. In the warning blocks only, a $100 \mathrm{~ms}$ warning tone was presented $800 \mathrm{~ms}$ after the fixation light appeared. In the gap condition, the fixation light was extinguished simultaneously with the offset of the warning tone, ${ }^{1}$ and following a $200-300 \mathrm{~ms}$ dark interval, either the right or the left LED was illuminated for $100 \mathrm{~ms}$. In the overlap condition, the target was presented at intervals varying between 200-300 ms after the offset of the warning tone, but the fixation light remained on until $600 \mathrm{~ms}$ after the offset of the target. The subjects were instructed to maintain fixation until the peripheral target appeared. In the prosaccade condition, subjects were instructed to look at the eccentric target. In the antisaccade condition subjects were instructed to make an eye movement the same dis-

\footnotetext{
${ }^{1}$ Fischer and Weber (1992) suggest that our use of a warning tone can produce an "overlap effect" which presumably would reduce the size of the gap effect relative to a warning absent condition. We point out, however, that the present design follows our previous work by making the tone offset concurrent with fixation offset so that no stimulus is present during the gap
}

tance but opposite in direction from the target light. In all cases, subjects were encouraged to respond as quickly as possible when the target appeared.

All blocks began with a calibration procedure that required subjects to fixate on each of the three LEDs in turn while eye position was sampled and saved for subsequent analyses.

\section{Data analysis}

Saccade onset was detected using a velocity criterion. The latency was taken to be the time at which the eye movement attained a velocity of $50 \mathrm{deg} / \mathrm{s}$, providing that this velocity was maintained for at least three consecutive samples. Saccades were detected automatically by the data analysis software and were verified by the experimenter. Responses with latencies less than $80 \mathrm{~ms}$ were eliminated as anticipations (Kalesnykas and Hallett 1987). Trials with blinks occurring within $500 \mathrm{~ms}$ prior to the target onset were eliminated. Direction errors were analyzed separately from RTs associated with correct responses.

\section{Results}

The latency data were analyzed with a three-way $\mathrm{AN}$ OVA with response condition (antisaccades vs prosaccades), fixation condition (gap vs overlap) and warning condition (presence vs absence of warning tone) as repeated factors. Three main effects and two significant interactions emerged. A main effect of response condition $\left(F_{19}=7.56, P<0.03\right)$ indicates that prosaccades were significantly faster overall than antisaccades (208 and $251 \mathrm{~ms}$ respectively). The acoustic warning signal had a robust effect on performance $\left(F_{1,9}=7.22, P<0.03\right)$ pro- 
Fig. 2 Magnitude of gap effect (overlap RT minus gap RT) for pro- and antisaccades with and without a warning signal
Table 1 Mean saccadic latency and standard errors

\section{GAP EFFECT : OVERLAP RT minus GAP RT}

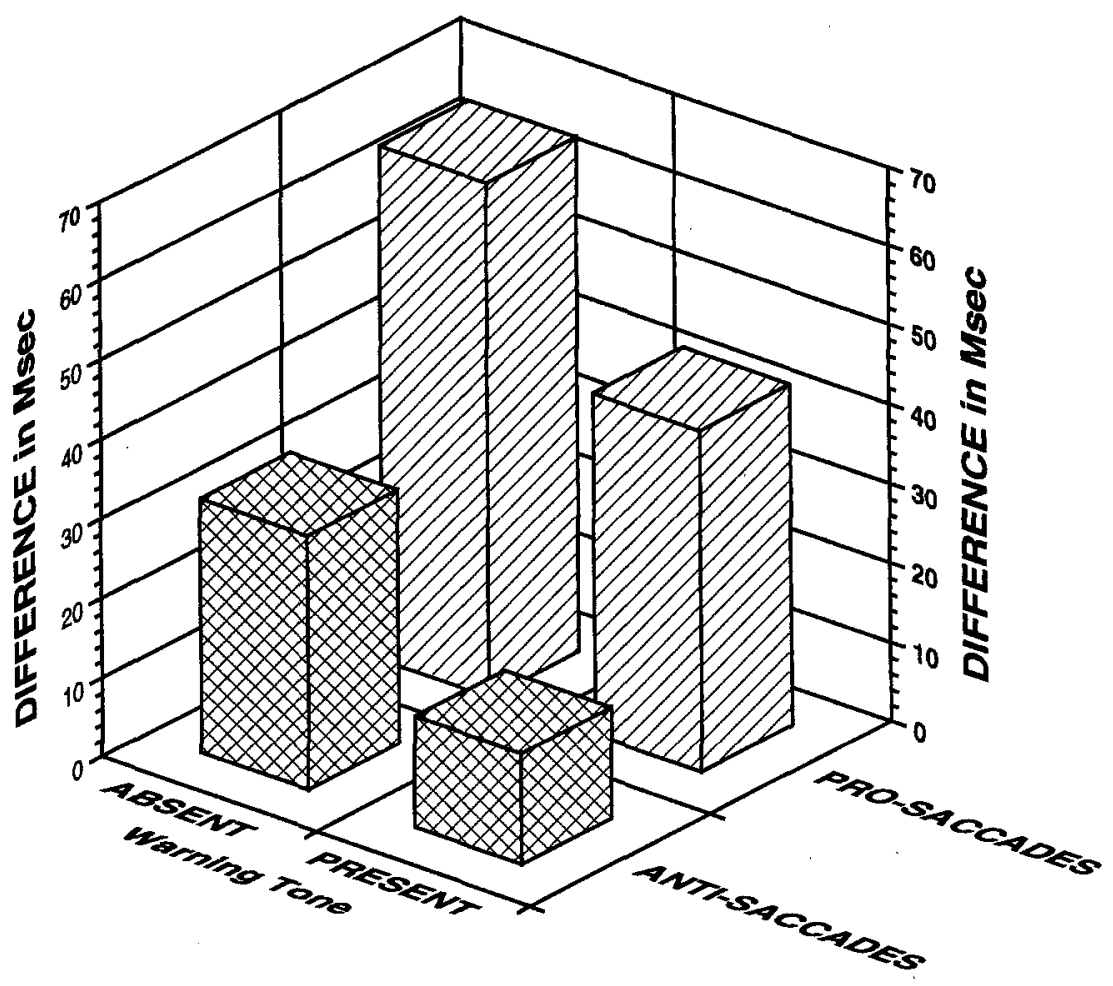

\begin{tabular}{|c|c|c|c|c|c|c|}
\hline & \multicolumn{3}{|c|}{ No-warning } & \multicolumn{3}{|l|}{ Warning } \\
\hline & Overlap & Gap & Gap effect & Overlap & Gap & Gap effect \\
\hline Prosaccades & $\begin{array}{r}262.30 \\
51.95\end{array}$ & $\begin{array}{r}197.06 \\
37.23\end{array}$ & 65 & $\begin{array}{r}230.19 \\
30.67\end{array}$ & $\begin{array}{r}187.17 \\
28.09\end{array}$ & 43 \\
\hline Antisaccades & $\begin{array}{r}312.25 \\
77.52\end{array}$ & $\begin{array}{r}280.51 \\
92.38\end{array}$ & 32 & $\begin{array}{r}258.07 \\
23.99\end{array}$ & $\begin{array}{r}243.69 \\
30.24\end{array}$ & 14 \\
\hline
\end{tabular}

ducing responses that were $33 \mathrm{~ms}$ faster on average than those generated in the absence of a warning signal ( 230 and $263 \mathrm{~ms}$ respectively). The lack of an interaction between response condition and warning signal indicates that the effect of this signal was equivalent for pro- and antisaccades $\left(F_{1,9}=0.915, P>0.3\right)$. The effect of fixation condition was highly significant $\left(F_{1,9}=58.78, P<0.0001\right)$ indicating that fixation offsets facilitated saccadic latencies. However, a significant interaction between response condition and fixation condition reflects the fact that prosaccades were associated with a greater gap effect than antisaccades $\left(F_{1,9}=11.31, P<0.008\right)$. Moreover, an interaction of fixation and warning signal conditions $\left(F_{1,9}=23.14, P<0.03\right)$ indicates that the size of the gap effect also depended upon whether or not a warning signal was present. In particular, the effect of fixation point offset was nearly twice as large in the absence of a concurrent warning signal than in its presence. This is exactly the outcome expected if one of the potential effects of extinguishing the fixation stimulus is to warn the subject of the imminent target.
These effects are illustrated in Fig. 2 which shows the size of the gap effect for pro- and antisaccades with and without warning signals (see Table 1 for mean RTs). Dunn's procedure for multiple, planned comparisons (Kirk 1968) was used to evaluate the reliability of the differences between the gap and overlap means (i.e., the gap effect). A strong gap effect is evident for prosaccades regardless of whether or not the warning signal occurs $(P<0.01)$. That is, for prosaccades a highly significant $43 \mathrm{~ms}$ gap effect is evident even with a warning signal. In contrast, for antisaccades a $32 \mathrm{~ms}$ gap effect without a warning signal $(P<0.01)$ is reduced to a non-significant $14 \mathrm{~ms}$ in the warning present condition $(P>0.05)$. This indicates that for antisaccades most of the effect of fixation point offset is due to its value as a warning signal. The magnitudes of the gap effect obtained for proand antisaccades with a warning signal present are nearly identical to those reported by Reuter-Lorenz et al. (1991) under similar experimental conditions ( $38 \mathrm{~ms}$ and $12 \mathrm{~ms}$, respectively). 
Fig. 3 Frequency histograms for subject A.J., the only one of four subjects showing responses meeting the dual latency and bimodality criteria for express saccades. Note the rarity of express saccades in the antisaccade condition. Saccades with latencies less than $80 \mathrm{~ms}$ are displayed in this figure, but were excluded from the analyses of mean latencies
Prosaccades: Gap with warning

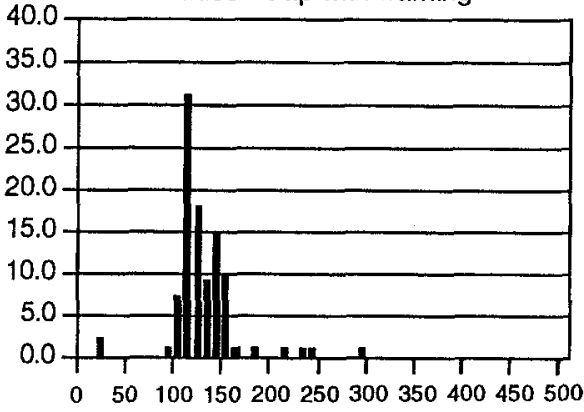

Antisaccades: Gap with warning

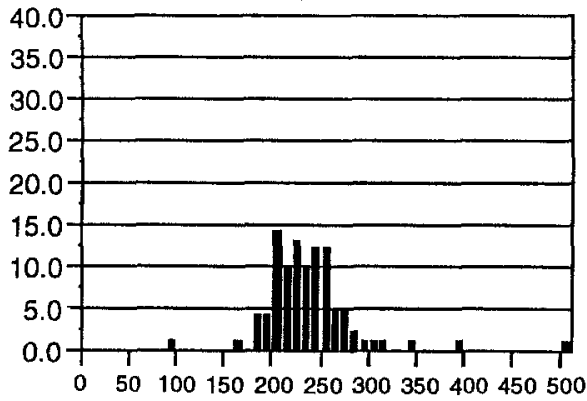

Prosaccades: Overlap with warning
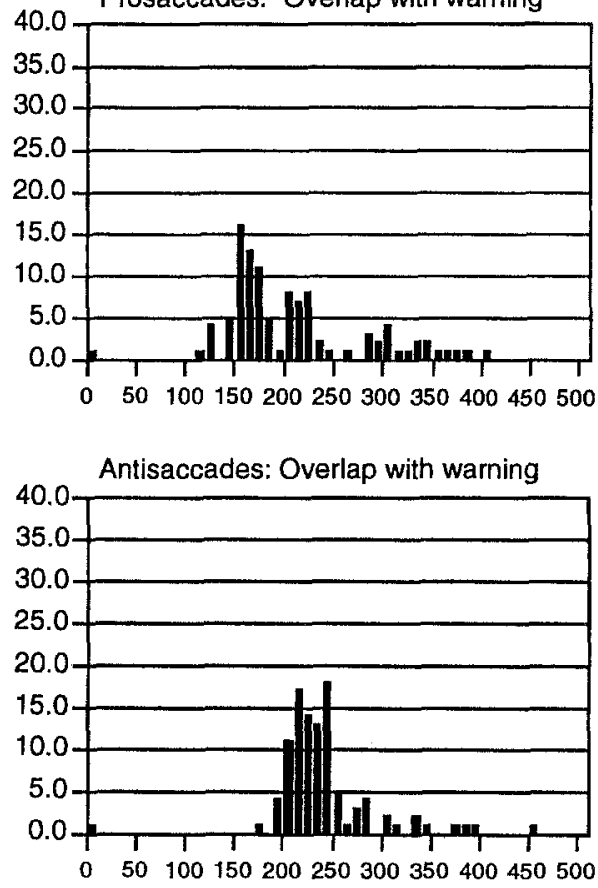

Distributional analyses

To evaluate the occurrence of express saccades, four of the subjects (L.L.B., A.J., H.M.O., and P.R.L.) participated in three additional trial blocks for each experimental condition. This yielded approximately 200 responses (100 left and 100 right saccades) per subject for both gap and overlap trials in each response-type-by-warning-condition combination. Frequency histograms were generated and inspected for the occurrence of bimodal or multimodal distributions of saccades with modes in the range of $100-120 \mathrm{~ms}$.

Only one of the four subjects showed clear evidence of responses that meet the dual criteria for express saccades: an early mode occurring around $100-120 \mathrm{~ms}$ in a bimodal or multimodal latency distribution. This subject showed express saccades in both the gap and overlap conditions. It is clear from visual inspection, however, that the frequency of express saccades is greater in the gap condition. Figure 3 illustrates this effect. As the only subject who shows express prosaccades, A.J. is an interesting case to examine for the existence of express saccades in the antisaccade task. In the gap condition only two responses have express latencies. The remaining saccades do not qualify as express. This confirms the pattern reported by Fischer and Weber (1992, 1993): antisaccades are rarely of the express type. The pattern is also consistent with the specific effect of fixation offsets on the mean latency of antisaccades revealed when warning effects are controlled: the gap effect for antisaccades is minimal.

It is important to note, however, that only one of the four subjects displayed evidence of express saccades even in the prosaccade condition. The others showed nu- merous bimodal or multimodal distributions, but multiple peaks were as likely in the overlap as in the gap conditions and, in these cases, were never associated with saccades in the $100 \mathrm{~ms}$ range.

\section{Direction errors and catch trial responses}

Direction errors were generally rare: the average across all conditions was less than $2.0 \%$. Warning signal presence/absence had no effect on the frequency of direction errors. Not surprisingly, direction errors were more frequent in antisaccade than prosaccade blocks $(2.36 \%$ and $0.44 \%$, respectively). For both types of responses, errors were more frequent in gap than overlap trials $(1.97 \%$ and $0.83 \%$, respectively). In the case of prosaccades, direction errors tended to be anticipatory responses with RTs less than $80 \mathrm{~ms}$. This was not generally true of errors in antisaccade blocks. In this case, the mean RTs approximated those associated with correct prosaccades in the gap and overlap conditions (184 and $247 \mathrm{~ms}$, respectively).

Responses on catch trials were also rare occurring on less than $3 \%$ of the catch trials, regardless of response type or warning signal presence/absence. Such responses were slightly more frequent on gap than on overlap trials (2.95\% vs $1.87 \%$, respectively).

\section{Discussion}

The present results indicate that the magnitude of the gap effect depends upon the presence or absence of an acoustic warning signal. Equating the warning information in gap and overlap conditions by including an acoustic 
warning signal reduces the size of the gap effect. This result indicates clearly that fixation offsets can serve as a warning signal and can speed performance by increasing response readiness. The data therefore corroborate the results reported by Ross and Ross (1980) and support the Kingstone and Klein (1993a) view of a response readiness component of fixation offsets.

The data also demonstrate that fixation offsets serve more than just a warning function. With this warning effect controlled, a $43 \mathrm{~ms}$ reduction in saccade latency still emerged for prosaccades. In contrast, for antisaccades the remaining gap effect was only $14 \mathrm{~ms}$. This pattern replicates the results of Reuter-Lorenz et al. (1991) and reveals the specific effects of "fixation release" or "disengaged fixation" on oculomotor performance (Fendrich et al. 1991; Kingstone and Klein 1993a; Tam and Stelmach 1993; Nozawa et al. 1995). Following the work of Munoz and Wurtz (1992), Reuter-Lorenz et al. (1991) suggested that active fixation inhibits collicular-based premotor processes involved in saccade generation and that the gap effect reflects a release from this inhibition. Since the superior colliculus (SC) presumably plays a greater role in prosaccades than antisaccades or manual responses, it makes sense that the effect of fixation offsets should be greatest for target-directed saccades.

The results of this study corroborate the Kingstone and Klein hypothesis that the gap effect may include both a general warning component and a fixation release component. Moreover, these results have an important implication for investigations of the gap effect: If the gap effect is to serve as an index of the time course of processes that are unique to the oculomotor system, then it is essential that the non-specific effects of fixation point offsets be controlled. In the absence of such control, the present data indicate that antisaccades, and presumably manual responses may show a robust gap effect. ${ }^{2}$ The warning component of fixation offsets rather than fixation release may indeed be driving such effects. Such non-specificity of the gap effect led Fischer and Weber (1993) to conclude that mean RT is an inadequate measure of reflex-like oculomotor behavior and that only the incidence of express saccades (defined by a distinct early mode in the RT distribution) provides the appropriate index. However, given the difficulties defining express saccades in terms of a unique early mode in the latency distribution, it is valuable to demonstrate that an alternative reliable and robust index exists.

A small gap effect for antisaccades emerged in the present experiment and in our previous report (ReuterLorenz et al. 1991). While this effect did not reach conventional levels of significance in either study, the fact

\footnotetext{
2 Iwasaki (1990) reported a gap effect of approximately $20 \mathrm{~ms}$ for choice manual responses in a paradigm which sounded a warning signal at least $2 \mathrm{~s}$ before target onset. This foreperiod is well beyond the range for optimal alerting effects, which means that the gap effect Iwasaki reports for manual RTs may well be due to the warning value of fixation offsets. Tam and Stelmach (1993) came to a similar conclusion when interpreting the gap effect they found for choice manual responses in the absence of an explicit warning signal
}

that it replicated is noteworthy. Therefore, we will consider a few possible explanations of this effect.

Saslow (1967) originally suggested that the gap effect was due to a greater likelihood of microsaccades in the overlap relative to the gap condition. Thus, the refractory period which followed microsaccades would delay responses more frequently in the overlap condition. This hypothesis predicts that the effects of fixation point offsets should be equivalent for pro- and antisaccades, which is clearly not the case. Moreover, recent work by Kingstone, Fendrich, Wessinger and Reuter-Lorenz (1995) found that microsaccades rarely occur during the interval between fixation offset and target onset. Furthermore, eliminating trials with microsaccades did not influence the magnitude of the gap effect.

It is possible that there is still some residual warning effect of fixation offset that is not entirely controlled for by the acoustic warning signal. That is, the combination of extinguishing the fixation point and presenting an acoustic warning signal may provide a more effective warning signal than the acoustic signal presented alone. We cannot rule out this possibility, but we emphasize that minimizing the warning signal value of fixation point offsets is an important procedure that can reveal the specificity of the gap effect.

Alternatively, the small gap effect for antisaccades may indicate that fixation release is a component of the processing architectures of antisaccades and possibly other oculomotor behaviors. ${ }^{3}$ The generation of antisaccades clearly depends on the integrity of the frontal eye fields (FEF), since patients with lesions to this area are impaired on this task (Guitton et al. 1985). Microstimulation studies indicate that the threshold current intensity needed to evoke electrically a saccade from the FEF is increased when the monkey is actively fixating (Goldberg et al. 1986) suggesting that saccades initiated by the FEF are also inhibited by active fixation. Although the FEF and SC appear to represent parallel channels for saccadic commands (Schiller et al. 1980), the FEFs project bilaterally to the SC. This latter pathway provides a possible route by which collicular mechanisms might influence saccades initiated at the level of the cortex [see Hughes et al. (1992) for review]. Thus, the inhibitory effects on the FEF observed by Goldberg et al. (1986) could also depend on processes within the SC.

Of course, the neural processes underlying fixation release could also influence sites other than the SC, such as oculomotor centers in the brainstem. In this case, all oculomotor responses would be expected to be influenced to some extent by this process. Why, however, would the facilitatory effects of fixation offsets be so much greater for prosaccades than antisaccades? It is likely that the generation of an antisaccade involves a number of component processes that are not involved in

\footnotetext{
3 While this manuscript was under review, Tam and Ono (1994) reported a gap effect for vergence eye movements. Although warning effects could have contributed to the $80 \mathrm{~ms}$ gap effect they report, their data suggest that fixation release may affect a variety of oculomotor behaviors
} 
the more natural prosaccade response. If we were to assume that (1) these processes are arranged in parallel with fixation-related processes, and (2) they must all be completed before a saccade occurs, and that (3) their time course is slow relative to fixation release (antisaccades do have long latencies), then we would actually expect that fixation offsets would not appreciably influence antisaccade latencies, for the simple reason that the overall latency of this type of parallel network is determined by the slowest of the component processes (Townsend and Ashby 1983). Thus, fixation release could occur with antisaccades, but the effect is masked by other, slower processes that also must run to completion before the (anti)saccade occurs (see also Nozawa et al. 1995).

In summary, the present results indicate that, in the absence of an explicit warning signal, fixation point offsets have more generalized facilitatory effects, presumably because of the alerting or warning signal value they can serve. However, if these non-specific effects are controlled, a strong gap effect is still observed for prosaccades whereas the effect for antisaccades is minimal. This selectivity suggests that the inhibitory effects of active fixation may be more closely linked with some oculomotor behaviors than with others. Thus the gap effect can provide a reliable behavioral index of a process that appears designed to modulate the interplay of active fixation and the generation of eye movement responses.

Acknowledgements This research was supported by the McDonnell-Pew program in Cognitive Neuroscience and by the Office of the Vice-President for Research, University of Michigan. We thank Amishi Jha and Jessica Small for their assistance with this project. We also thank Alan Kingstone and two anonymous reviewers for their comments on this report.

\section{References}

Fendrich R, Hughes HC, Reuter-Lorenz PA (1991) Fixation point offsets reduce the latency of saccades to acoustic targets. Percept Psychophys 50:383-387

Fischer B, Boch R (1983) Saccadic eye movements after extremely short reaction times in the monkey. Exp Brain Res 260: $21-26$

Fischer B, Breitmeyer B (1987) Mechanisms of visual attention revealed by saccadic eye movements. Neuropsychologia 25: 73-83

Fischer B, Ramsperger E (1984) Human express saccades:extremely short reaction times of goal directed eye movements. Exp Brain Res 57:191-195

Fischer B, Ramsperger E (1986) Human express-saccades:effects of randomization and daily practice. Exp Brain Res 64: $569-578$

Fischer B, Weber H (1992) Characteristics of "anti" saccades in man. Exp Brain Res 89:415-424

Fischer B, Weber H (1993) Express saccades and visual attention. Behav Brain Sci 16:553-610

Fischer B, Weber H, Biscaldi M, Aiple F, Otto P, Stuhr V (1993) Separate population of visually guited saccades in humans: Reaction times and amplitudes. Exp Brain Res 92:528541
Goldberg ME, Bushnell MC, Bruce CJ (1986) The effect of attentive fixation on eye movements evoked by electrical stimulation of the frontal eye fields. Exp Brain Res 61:579-584

Guitton D, Buchtel HA, Douglas RM (1985) Frontal lobe lesions in man cause difficulties in suppressing reflexive glances and in generating goal-directed saccades. Exp Brain Res 58: $455-472$

Hughes HC, Reuter-Lorenz PA, Fendrich R, Gazzaniga MS (1992) Bidirectional control of saccadic eye movements by the disconnected cerebral hemispheres. Exp Brain Res 91:335-339

Iwasaki S (1990) Facilitation of reaction times with GAP paradigm: comparison of manual and saccadic responses. Ergonomics 33:833-850

Kalesnykas R, Hallett PE (1987) The differentiation of visually guided and anticipatory saccades in gap and overlap paradigms. Exp Brain Res 68:115-121

Kingstone A, Fendrich R, Wessinger CM, Reuter-Lorenz PA (1995) Are microsaccades responsible for the gap effect? Percept Psychs

Kingstone A, Klein R (1990) Attention and express saccades. Bull Psychon Soc 28:512

Kingstone A, Klein RM (1993a) Visual offsets facilitate saccadic latency: does predisengagement of visuospatial attention mediate this gap effect? J Exp Psych: Human Percept Perf 19:1251-1265

Kingstone A, Klein RM (1993b) What are human express saccades? Percept Psychophys 54:260-273

Kirk RE (1968) Experimental design: procedures for the behavioral sciences. Wadsworth, Belmont, Calif

Klein RM, Kingstone AF (1993) Why do visual offsets reduce saccadic latencies? Behav Brain Sci 16:583-584

Munoz DP, Wurtz RH (1992) Role of the rostral superior colliculus in active visual fixation and execution of express saccades. J Neurophysiol 67:1000-1102

Nozawa G, Reuter-Lorenz PA, Hughes HC (1995) Parallel and serial processes in the human oculomotor system: bimodal integration and express saccades. Biol Cybern

Reuter-Lorenz PA, Hughes HC (1993) Warning signals, response specificity and the gap effect: implications for a non-attentional account. Behav Brain Sci 16:585-586

Reuter-Lorenz PA, Hughes HC, Fendrich R (1991) The reduction of saccadic latency by prior offset of the fixation point: an analysis of the "gap effect". Percept Psychophys 49: 167-175

Ross LE, Ross SM (1980) Saccade latency and warning signals: stimulus onset, offset and change as warning events. Percept Psychophys 27:251-257

Ross SM, Ross LE (1981) Saccade latency and warning signals: Effects of auditory and visual stimulus onset and offset. Percept Psychophys 29:429-437

Saslow MG (1967) Effects of components of displacement step stimuli upon latency for saccadic eye movements. J Opt Soc Am 57:1024-1029

Schiller PH, True SD, Conway JL (1980) Deficits in eye movements following frontal eye-field and superior colliculus ablations. J Neurophysiol 44:1175-1189

Schiller PH, Sandell JH, Maunsell JHR (1987) The effect of frontal eye field and superior colliculus lesions on saccadic latencies in the rhesus monkey. $J$ Neurophysiol 57:1033-1049

Tam WJ, Ono H (1994) Fixation disengagement and eye-movement latency. Percept Psychophys 56:251-260

Tam WJ, Stelmach LB (1993) Viewing behavior: ocular and attentional disengagement. Percept Psychophys 56:251-260

Townsend JT, Ashby FG (1983) Stochastic modeling of elementary psychological processes. Cambridge University Press, Cambridge, UK

Wenban-Smith MG, Findlay J M (1991) Express saccades: is there a separate population in humans? Exp Brain Res 87:218-222 